\title{
BMJ Open Migration and head penetration of Vitamin-E diffused cemented polyethylene cup compared to standard cemented cup in total hip arthroplasty: study protocol for a randomised, double-blind, controlled trial (E1 HIP)
}

\author{
Olof Sköldenberg, Agata Rysinska, Ghazi Chammout, Mats Salemyr, \\ Olle Muren, Henrik Bodén, Thomas Eisler
}

To cite: Sköldenberg 0 , Rysinska A, Chammout G, et al. Migration and head penetration of Vitamin-E diffused cemented polyethylene cup compared to standard cemented cup in total hip arthroplasty: study protocol for a randomised, double-blind, controlled trial (E1 HIP). BMJ Open 2016;6: e010781. doi:10.1136/ bmjopen-2015-010781

- Prepublication history for this paper is available online To view these files please visit the journal online (http://dx.doi.org/10.1136/ bmjopen-2015-010781).

Received 7 December 2015 Revised 12 April 2016 Accepted 26 April 2016

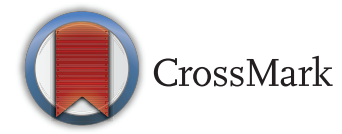

Department of Clinical Sciences, Danderyd Hospital, Karolinska Institutet, Stockholm, Sweden

Correspondence to Dr Olof Sköldenberg; olof.skoldenberg@gmail.com

\section{ABSTRACT}

Introduction: In vitro, Vitamin-E-diffused, highly crosslinked polyethylene (PE) has been shown to have superior wear resistance and improved mechanical properties when compared to those of standard highly cross-linked PE liners used in total hip arthroplasty (THA). The aim of the study is to evaluate the safety of a new cemented acetabular cup with Vitamin-E-doped PE regarding migration, head penetration and clinical results. Methods and analysis: In this single-centre, doubleblinded, randomised controlled trial, we will include 50 patients with primary hip osteoarthritis scheduled for THA and randomise them in a 1:1 ratio to a cemented cup with either argon gas-sterilised PE (control group) or Vitamin-E-diffused PE (vitamin-e group). All patients and the assessor of the primary outcome will be blinded and the same uncemented stem will be used for all participants. The primary end point will be proximal migration of the cup at 2 years after surgery measured with radiostereometry. Secondary end points include proximal migration at other follow-ups, total migration, femoral head penetration, clinical outcome scores and hip-related complications. Patients will be followed up at 3 months and at 1,2, 5 and 10 years postoperatively. Results: Results will be analysed using $95 \% \mathrm{Cls}$ for the effect size. A regression model will also be used to adjust for stratification factors.

Ethics and dissemination: The ethical committee at Karolinska Institutet has approved the study. The first results from the study will be disseminated to the medical community via presentations and publications in relevant medical journals when the last patient included has been followed up for 2 years.

Trial registration number: NCT02254980.

\section{INTRODUCTION}

The major factor limiting the lifespan of a total hip arthroplasty (THA) is periprosthetic
Strengths and limitations of this study

Blinded assessment of primary outcome.

- Randomised controlled trial.

- High-precision measurement of primary outcome using radiostereometry.

- Proxy variable for loosening of implants.

- Blinding of surgeons not possible.

osteolysis and loosening secondary to wear of the ultrahigh molecular weight polyethylene (PE) of acetabular components. ${ }^{12}$ In vitro, Vitamin-E-doped highly cross-linked PE have been shown to have superior wear resistance and improved mechanical properties when compared to that of standard PE. ${ }^{3}$ The first randomised clinical trials on Vitamin-E PE in THA have recently been published by our research group and found a low wear rate up to 2 years postoperatively when compared to controls. ${ }^{4}$ Others have confirmed these findings $^{5}$ also for larger-diameter femoral heads. ${ }^{6}$ All of these trials use uncemented acetabular cups intended for biological fixation. In many countries, the most common fixation method for the acetabular component is bone cement ${ }^{1}$ and there are now on the market new acetabular components with Vitamin-E-doped PE intended for cemented fixation. Radiostereometry (RSA) can be used to predict loosening of THA implants in vivo and is the gold standard in evaluating new prosthetic implants. ${ }^{7} 8$ A proximal migration of $1.0 \mathrm{~mm}$ up to 2 years after surgery significantly increases the risk of revision for acetabular cups ${ }^{9}$ and this threshold can be implemented in a phased, evidencebased introduction of new implants, ${ }^{10}$ since 
they allow early detection of high-risk cups while exposing a small number of patients. ${ }^{7-9}$

The aim of the study is to evaluate the safety of a new acetabular cup with Vitamin-E-doped PE by comparing it to a clinically well-proven cup with standard PE regarding migration, head penetration and clinical results. We hypothesised that the new Vitamin-E PE is non-inferior to standard PE in terms of early (2 years) migration of the cup.

\section{PATIENTS AND METHODS}

\section{Setting and design}

A single-centre, randomised, double-blinded, controlled trial will be carried out from 2013 to 2025 at the Orthopaedic Department of Danderyd Hospital, Stockholm, in collaboration with the Karolinska Institute, Stockholm. The Ethics Committee of the Karolinska Institute has approved the study (No. 2011/ 2003-31/1). The guidelines of Good Clinical Practice (GCP-ICH) will be followed. ${ }^{11}$ The trial is initiated, designed and performed as an academic investigation and registered at ClinicalTrials.gov (NCT02254980). The guidelines of the CONSORT Statement will be followed $^{12}$ for the final paper and the SPIRIT guidelines for the study protocol. ${ }^{13}$

\section{Randomisation and blinding}

Patients will be randomised in a 1:1 ratio to the control group or vitamin-e group using concealed envelopes. A randomly assigned batch size of 4-10 (in increments of 2 ; thus $4,6,8$ or 10) will be used. We will use sex and age ( $<65$ and $\geq 65$ years) as stratification factors to ensure that the baseline characteristics are similar in the two groups. The patients and staff will be blinded to treatment. Since $\alpha$-tocopherol taints PE yellow, the surgeons cannot be blinded to allocation. The outcome assessor of the migration primary and secondary end points will be blinded when performing the RSA analysis. The patients who are blinded fill out all forms for the clinical outcome scores.

\section{Patients}

Consecutive patients aged 40-75 years who are being planned for THA will be eligible for inclusion in the study. We will include patients with a primary osteoarthritis of the hip and a willingness and ability to follow the study protocol. We will exclude patients with inflammatory arthritis or secondary osteoarthritis, with a femoral or pelvic anatomy after hip dysplasia not suitable for implantation of components, those who have ongoing oestrogen treatment or treatment with bisphosphonates, cortisol or cytostatic drugs 6 months prior to surgery and those who are not suited for the study for other reasons (for instance, substance abuse).

\section{Surgery and allocation}

The randomisation will allocate to THA with the cemented Muller Exceed ABT cup (Biomet, Warsaw, Indiana,
USA) acetabular component with either VitaminE-diffused PE (vitamin-e group, E1) or argon gassterilised compression moulded PE (control group, Arcom PE). A standard posterior approach with repair of the posterior capsule and external rotators will be used. The femoral component will be an uncemented, tapered, proximally porous-coated and hydroxyapatitecoated stem composed of a Ti-6Al-4V titanium alloy (Bi-Metric HA; Biomet) and a $32 \mathrm{~mm}$ chromium-cobalt head. The surgical technique as described by the manufacturers for the implants will be followed. Thirdgeneration cementation technique will be used. We have a long experience of using the Muller cup and Bi-Metric stem, so no learning curve will be expected. ${ }^{14}$ Intravenous tranexamic acid (Cyclokapron; Pfizer, Sollentuna, Sweden) will be administered before the start of surgery to reduce bleeding. Prophylactic antibiotics (cloxacillin; Meda, Solna, Sweden) will be administered $30 \mathrm{~min}$ preoperatively and 24 hours postoperatively, and dalteparin (Fragmin; AstraZeneca, Sodertalje, Sweden) for 10 days postoperatively to prevent thrombosis. Patients will start rehabilitation on the first postoperative day. All patients will mobilise with full weight-bearing, under supervision of a physiotherapist, with the use of suitable walking aids during the first 6 weeks.

\section{End points and follow-ups}

The primary end point variable will be proximal migration of the cup at 2 years, measured with RSA. This end point was chosen since every millimetre increase in 2-year proximal migration has been verified to increase the revision rate of an acetabular implant by $10 \%$ at 10 years. ${ }^{9}$ This predictive power of early migration on future revision is widely used in RSA studies and is the reason the method is the gold standard in evaluating new implants in joint arthroplasty. ${ }^{7-9} 15$ The secondary end points will include proximal migration at all other follow-ups, maximum total point motion (MTPM) of the cup, head penetration of the prosthetic head into the cup, migration of the femoral stem, development of radiolucent lines between bone and cement around the cup, functional outcome scores, serological markers of inflammation and hip-related complications up to 10 years. Follow-up will be done at inclusion and at 3 months and 1, 2, 5 and 10 years postoperatively (figure 1) with the primary end point evaluated at 2 years.

\section{RSA and radiological evaluation}

RSA is a high-precision method of assessing threedimensional (3D) micromotion from calibrated stereo radiographs and is used for evaluating new implants since early migration can predict loosening. ${ }^{7}{ }^{8}$ Nine Tantalum markers $(1.0 \mathrm{~mm})$ will be put in the pelvis surrounding the cup and the surgeon will fix nine tantalum markers in the implant before cementing. We will follow the published guidelines for RSA. ${ }^{15}$ We will use digital 


\begin{tabular}{|c|c|c|c|c|c|c|c|c|c|c|}
\hline & $<2 \mathrm{~m}$ & $-2 v$ & $\mathbf{0}$ & $+1 d$ & 5d & $3 \mathrm{~m}$. & $1 \mathrm{y}$ & $2 y$ & $5 y$ & $10 \mathrm{y}$ \\
\hline & Screening & Inclusion & $\begin{array}{c}\text { Randomisation } \\
\text { and surgery }\end{array}$ & P0D1 & $\begin{array}{c}\text { End of } \\
\text { In-pt } \\
\text { stay }\end{array}$ & $\begin{array}{c}\text { Follow- } \\
\text { up }\end{array}$ & $\begin{array}{c}\text { Follow- } \\
\text { up }\end{array}$ & $\begin{array}{c}\text { Follow- } \\
\text { up }\end{array}$ & $\begin{array}{c}\text { Follow- } \\
\text { up }\end{array}$ & $\begin{array}{l}\text { Follow- } \\
\text { up }\end{array}$ \\
\hline Screening for eligibility & $\mathrm{x}$ & & & & & & & & & \\
\hline Informed consent & & $\mathrm{x}$ & & & & & & & & \\
\hline Baseline data & & $\mathrm{x}$ & & & & & & & & \\
\hline Randomization & & & $\mathrm{x}$ & & & & & & & \\
\hline X-ray & & $\mathrm{x}$ & & $\mathrm{x}$ & & & $\mathrm{x}$ & $\mathrm{x}$ & $\mathrm{x}$ & $\mathrm{x}$ \\
\hline RSA & & & & $\mathrm{x}$ & & $\mathrm{x}$ & $\mathrm{x}$ & $\mathrm{x}$ & $\mathrm{x}$ & $\mathrm{x}$ \\
\hline $\mathrm{HHS}$ & & $\mathrm{x}$ & & & & & $\mathrm{x}$ & $\mathrm{x}$ & $\mathrm{x}$ & $\mathrm{x}$ \\
\hline HOOS & & $\mathrm{x}$ & & & & & $\mathrm{x}$ & $\mathrm{x}$ & $\mathrm{x}$ & $\mathrm{x}$ \\
\hline VAS & & $\mathrm{x}$ & & & & & $\mathrm{x}$ & $\mathrm{x}$ & $\mathrm{x}$ & $\mathrm{x}$ \\
\hline EQ-5D & & $\mathrm{x}$ & & & & & $\mathrm{x}$ & $\mathrm{x}$ & $\mathrm{x}$ & $\mathrm{x}$ \\
\hline Adverse events & & & $\mathrm{x}$ & $\mathrm{x}$ & $\mathrm{x}$ & $\mathrm{x}$ & $\mathrm{x}$ & $\mathrm{x}$ & $\mathrm{x}$ & $\mathrm{x}$ \\
\hline Blood test & & $\mathrm{x}$ & & $\mathrm{x}$ & & $\mathrm{x}$ & $\mathrm{x}$ & $\mathrm{x}$ & $\mathrm{x}$ & $x$ \\
\hline
\end{tabular}

Figure 1 Blood test, serum C-terminal telopeptide of type I collagen (SCTx), pyridinoline cross-linked carboxyterminal telopeptide of type I collagen (1CTP), C reactive protein (CRP), IL-6, Hb; EQ-5D, quality of life score; HHS, Harris hip score; HOOS, Hip Osteoarthritis Outcome Score; m, month; RSA, radiostereometry; v, week; VAS, visual analogue scale for hip pain; $\mathrm{X}$-ray, anterioposterior and lateral radiographs; $\mathrm{y}$, year.

calibrated radiographs, a uniplanar calibration cage (Uniplanar digital 43; RSA Biomedical AB) and analyse all data using the UmRSA software (RSA Biomedical AB, Umeå, Sweden). The markers in the acetabulum form one segment and the markers in the cup form another segment. The 3D translations and rotations of the calculated centre of gravity of the cup in relation to the acetabular bone segment will be calculated at each follow-up visit and compared with the immediate postoperative measurements. The proximal migration of the cup up to 2 years postoperatively, which has been found to be a clinically relevant end point for correlating RSA results to register data, ${ }^{9}$ will be used for the primary end point. A migration threshold of $1.0 \mathrm{~mm}$ during the first 2 years will be used. ${ }^{9}$ The MTPM of the cup, which is the 3D translation vector of the marker in the implant that has the largest movement and is seen as an indicator of the overall magnitude of migration, will be used for the secondary end point of overall migration of the cups. The centre of the prosthetic head will also be measured with the built-in edge-detection technique of the software and used to measure head penetration into the PE and calculate linear head penetration. The centre of the prosthetic head and its movement in correlation to the femoral stem will also be used to measure the migration of the stem. At 1 year, we will perform two examinations 15 min apart on all patients with complete repositioning of the X-ray tubes and the calibration cage. We will use these measurements to calculate the precision as the 99\% CI (SD 2.7) of the difference between the examinations. The mean error of rigid body fitting will be used to evaluate the stability of the markers over time. ${ }^{16} \mathrm{We}$ will exclude examinations in which this value is $>0.3 \mathrm{~mm}$ because this indicates migration of the markers. The condition number is used to evaluate the distribution of the markers and a high value precludes accurate measurements of z-translation as well as segment rotation and MTPM. Therefore, in examinations in which the condition number exceeded 150, only transverse (x) and vertical (y) translations will be calculated. ${ }^{16}$ In addition to the RSA evaluation, digital anteroposterior and lateral radiographs will be taken (Bucky Diagnostics; Philips, Eindhoven, the Netherlands). With these, we will evaluate the presence of radiolucent lines between the bone and the cement in the DeLee and Charnley ${ }^{17}$ zones around the cup. Heterotopic ossification will be evaluated according to the Brooker et $a l^{18}$ classification.

\section{Functional outcomes}

The functional outcome scores will be the Harris hip score $^{19}$ and the Hip disability and Osteoarthritis Outcome Score. ${ }^{20}{ }^{21}$ Both are valid and widely used for evaluating hip function after THA. Health-related quality of life will be assessed by the EQ-5D (EuroQoL). ${ }^{22}{ }^{23}$ EQ-5D uses five dimensions: mobility, self-care, usual activity, pain/discomfort and anxiety/depression. Other end points include pain in the operated hip evaluated with visual analogue scale.

\section{Hip-related complications and adverse events}

All hip-related complications will be recorded throughout the study period. To make sure that we capture all events, we will use the unique Swedish personal id number and collect data prospectively throughout the study period through a combination of a search of our surgical and medical databases, follow-up visits and the Swedish Hip Arthroplasty Register. Other non-hip-related adverse events (AEs) and serious AEs (SAEs) will also be collected throughout the study period.

\section{Serological markers}

As a secondary end point, serological markers of inflammatory response (high-sensitivity $\mathrm{C}$ reactive protein and interleukin 6) will be measured to investigate whether the Vitamin-E in the acetabular components reduces the inflammatory response during the study period. ${ }^{24}{ }^{25} \mathrm{We}$ will also measure the levels of serum C-terminal telopeptide of type I collagen and pyridinoline cross-linked carboxy-terminal telopeptide of type I collagen, both of 
which can be used as biomarkers in serum to measure the rate of bone turnover. ${ }^{26}$

\section{Data quality assurance}

Study progress and study conduct will be monitored before, during and after the study by an external monitor to ensure that GCP-ICH, ${ }^{11}$ regulatory requirements and all aspects of the protocol are followed. All study data will be collected and managed in a digital case report form (CRF) using Research Electronic Data Capture (REDCap) tools hosted at Karolinska Institutet. ${ }^{27}$ REDCap is a secure, web-based application designed to support data capture for research studies, providing: (1) an intuitive interface for validated data entry; (2) audit trails for tracking data manipulation and export procedures; (3) automated export procedures for seamless data downloads to common statistical packages; and (4) procedures for importing data from external sources. The medical records and other documents will be reviewed for verification of agreement with data on the CRF. The participant has a right for protection against invasion of privacy. In this study, each participant will receive a unique identification number, which will be linked to the CRF. The data will then be blinded correspondingly in all data analyses. However, the study monitor, auditor, representative from any regulatory authority and the appropriate Ethical Committee are permitted to review the participant's primary medical records including laboratory test result reports, ECG reports, admission and discharge summaries, and $\mathrm{AE}$ and SAE reports generated during the study period.

\section{Sample size}

The study is designed to show that the vitamin-e group, compared to the control group, has neither lower nor higher proximal migration (y-translation) than the clinically relevant migration threshold of $0.2 \mathrm{~mm} .{ }^{9} \mathrm{~A}$ noninferiority power analysis that with a power of $90 \%$ will show that the mean for the proximal migration at 2 years in the vitamin-e group is the same as the mean for the control group requires a sample size of 18 participants in each group. This assumes that both groups have a common within-group SD of 0.21, estimated from one of our previous studies, ${ }^{28}$ and that a difference of $0.2 \mathrm{~mm}$ or less is clinically irrelevant as reported in a recent meta-analysis. ${ }^{9}$ The $\alpha$ (two-tailed) is set at 0.05 . We will include 25 patients in each group (50 total) to allow for loss to follow-up and loss of data due to the technical nature of RSA.

\section{Analysis}

The analyses will be performed on the basis of the intention-to-treat principle, and all patients who are allocated to either group will be included in the analysis, regardless of the actual surgery performed. Descriptive statistics (means and SDs) will be used to describe the patient characteristics and outcome variables at the measurement points. We will use Student's t-test and
Levene's test for comparison of the end points with the 95\% CI presented. An analysis of covariance of the primary end point will also be used to reduce variance, adjusted for exposure variable (control group/vitamin-e group) and stratification factors (men/women and $<65$ / $\geq 65$ years). For participants who had withdrawn from the study before completion, the data from the last observation will be carried forward (imputed). The analyses will be performed with SPSS V.22.0 for Windows (SPSS, Chicago, Illinois, USA) statistical software.

\section{ETHICS AND DISSEMINATION}

The ethical committee at Karolinska Institutet has approved the study. The first results from the study will be disseminated to the medical community via presentations and publications in relevant medical journals when the last patient included has been followed up for 2 years. Further publications will be presented at 5 and 10 years.

Acknowledgements The authors gratefully acknowledge the support of our institution and department and most of all our research nurses Helene Sjöö, Paula Kelly-Pettersson, Marie Ax and Lise-Lotte Widmark.

Contributors OS conceived the original study and developed the protocol with $A R, G C, T E, O M, M S$ and $H B$. OS led the writing of the manuscript, with contributions from AR, GC, TE, OM, MS and HB. All authors contributed to the editing and redrafting.

Funding The study was supported by these foundations: Åke Wiberg stiftelse, Loo and Hans Ostermans Stiftelse, Sven Norén foundation and the regional agreement on medical training and clinical research (ALF) between Stockholm County Council and Karolinska Institutet. Biomet partially funded the RSA examinations in the study but has no further input or participation in the trial.

Competing interests None declared.

Ethics approval This study was approved by the Ethics committee at the Karolinska Institute, Stockholm, Sweden.

Provenance and peer review Not commissioned; externally peer reviewed.

Open Access This is an Open Access article distributed in accordance with the Creative Commons Attribution Non Commercial (CC BY-NC 4.0) license, which permits others to distribute, remix, adapt, build upon this work noncommercially, and license their derivative works on different terms, provided the original work is properly cited and the use is non-commercial. See: http:// creativecommons.org/licenses/by-nc/4.0/

\section{REFERENCES}

1. Garellick G, Kärrholm J, Rogmark C, et al. Swedish Hip Arthroplasty Register Annual Report 2011. 2011. http://www.shpr.se/Libraries/ Documents/\%C3\%85rsrapport_2011_avseende_verksamhets\%C3\% A5ret 2011.sflb.ashx (accessed 27 Oct 2011).

2. Harris WH. Wear and periprosthetic osteolysis: the problem. Clin Orthop Relat Res 2001;393:66-70.

3. Oral E, Christensen SD, Malhi AS, et al. Wear resistance and mechanical properties of highly cross-linked, ultrahigh-molecular weight polyethylene doped with vitamin E. J Arthroplasty 2006;21:580-91.

4. Salemyr M, Muren O, Ahl T, et al. Vitamin-E diffused highly cross-linked polyethylene liner compared to standard liners in total hip arthroplasty. A randomized, controlled trial. Int Orthop 2015;39:1499-505.

5. Shareghi B, Johanson PE, Karrholm J. Femoral head penetration of vitamin E-infused highly cross-linked polyethylene liners: a randomized radiostereometric study of seventy hips followed for two years. J Bone Joint Surg Am 2015;97:1366-71. 
6. Lindalen E, Nordsletten L, Høvik Ø, et al. E-vitamin infused highly cross-linked polyethylene: RSA results from a randomised controlled trial using $32 \mathrm{~mm}$ and $36 \mathrm{~mm}$ ceramic heads. Hip Int 2015;25:50-5.

7. Kärrholm J, Borssén B, Löwenhielm G, et al. Does early micromotion of femoral stem prostheses matter? 4-7-year stereoradiographic follow-up of 84 cemented prostheses. J Bone Joint Surg Br 1994;76:912-17.

8. Selvik G. Roentgen stereophotogrammetry. A method for the study of the kinematics of the skeletal system. Acta Orthop Scand Suppl 1989;232:1-51.

9. Pijls BG, Nieuwenhuijse MJ, Fiocco M, et al. Early proximal migration of cups is associated with late revision in THA: a systematic review and meta-analysis of 26 RSA studies and 49 survival studies. Acta Orthop 2012;83:583-91.

10. Malchau H, Bragdon CR, Muratoglu OK. The stepwise introduction of innovation into orthopedic surgery: the next level of dilemmas. $J$ Arthroplasty 2011;26:825-31.

11. Vijayananthan A, Nawawi O. The importance of Good Clinical Practice guidelines and its role in clinical trials. Biomed Imaging Interv J 2008;4:e5.

12. Schulz KF, Altman DG, Moher D, CONSORT Group. CONSORT 2010 statement: updated guidelines for reporting parallel group randomised trials. BMJ 2010;340:c332.

13. Chan AW, Tetzlaff JM, Altman DG, et al. SPIRIT 2013 statement: defining standard protocol items for clinical trials. Ann Intern Med 2013;158:200-7.

14. Boden H, Salemyr M, Sköldenberg O, et al. Total hip arthroplasty with an uncemented hydroxyapatite-coated tapered titanium stem: results at a minimum of 10 years' follow-up in 104 hips. J Orthop Sci 2006;11:175-9.

15. Valstar ER, Gill R, Ryd L, et al. Guidelines for standardization of radiostereometry (RSA) of implants. Acta Orthop 2005;76:563-72.

16. Söderkvist I, Wedin PA. On condition numbers and algorithms for determining a rigid-body movement. BIT 1994;34:424-36.

17. DeLee JG, Charnley J. Radiological demarcation of cemented sockets in total hip replacement. Clin Orthop Relat Res 1976 : 20-32.
18. Brooker AF, Bowerman JW, Robinson RA, et al. Ectopic ossification following total hip replacement. Incidence and a method of classification. J Bone Joint Surg Am 1973;55:1629-32.

19. Harris WH. Traumatic arthritis of the hip after dislocation and acetabular fractures: treatment by mold arthroplasty. An end-result study using a new method of result evaluation. $J$ Bone Joint Surg Am 1969;51:737-55.

20. Nilsdotter AK, Lohmander LS, Klässbo M, et al. Hip disability and osteoarthritis outcome score (HOOS)—validity and responsiveness in total hip replacement. BMC Musculoskelet Disord 2003;4:10.

21. Davis AM, Perruccio AV, Canizares M, et al. Comparative, validity and responsiveness of the HOOS-PS and KOOS-PS to the WOMAC physical function subscale in total joint replacement for osteoarthritis. Osteoarthr Cartil 2009;17:843-7.

22. Burström K, Johannesson M, Diderichsen F. Swedish population health-related quality of life results using the EQ-5D. Qual Life Res 2001;10:621-35.

23. Rabin R, de Charro F. EQ-5D: a measure of health status from the EuroQol Group. Ann Med 2001;33:337-43.

24. Chaganti RK, Purdue E, Sculco TP, et al. Elevation of serum tumor necrosis factor alpha in patients with periprosthetic osteolysis: a case-control study. Clin Orthop Relat Res 2014;472:584-9.

25. Bergin PF, Doppelt JD, Kephart CJ, et al. Comparison of minimally invasive direct anterior versus posterior total hip arthroplasty based on inflammation and muscle damage markers. J Bone Joint Surg Am 2011;93:1392-8.

26. Rosen HN, Moses AC, Garber J, et al. Serum CTX: a new marker of bone resorption that shows treatment effect more often than other markers because of low coefficient of variability and large changes with bisphosphonate therapy. Calcif Tissue Int 2000;66: 100-3.

27. Harris PA, Taylor R, Thielke R, et al. Research electronic data capture (REDCap)-a metadata-driven methodology and workflow process for providing translational research informatics support. J Biomed Inform 2009;42:377-81.

28. Salemyr M, Muren O, Eisler T, et al. Porous titanium construct cup compared to porous coated titanium cup in total hip arthroplasty. A randomised controlled trial. Int Orthop 2015;39:823-32. 